\title{
X-RAY DETERMINATION OF THE BLACK-HOLE MASS IN CYGNUS X-1
}

\author{
A.KUBOTA AND K.MAKISHIMA \\ Department of Physics, University of Tokyo \\ 7-3-1, Hongo, Bunkyo-ku, Tokyo 113 JAPAN \\ T. DOTANI, H. INOUE, K. MITSUDA, F. NAGASE, H. NEGORO AND \\ Y. UEDA \\ Institute of Space and Astronautical Science, Sagamihara, Kanagawa \\ 229, JAPAN \\ K. EBISAWA \\ Universities Space Research Association, NASA/GSFC \\ S. KITAMOTO \\ Department of Earth and Space Science, Osaka Univ.Osaka \\ $560, J A P A N$ \\ AND \\ Y. TANAKA \\ SRON-Utrecht, Sorbonnelaan 2, 3584 CA Utrecht, the Netherlands
}

About $10 \mathrm{X}$-ray binaries in our Galaxy and LMC/SMC are considered to contain black hole candidates (BHCs). Among these objects, Cyg X-1 was identified as the first $\mathrm{BHC}$, and it has led $\mathrm{BHCs}$ for more than 25 years(Oda 1977,Liang and Nolan 1984). It is a binary system composed of normal blue supergiant star and the $\mathrm{X}$-ray emitting compact object. The orbital kinematics derived from optical observations indicates that the compact object is heavier than $\sim 4.8 \mathrm{M}_{\odot}$ (Herrero 1995), which well exceeds the upper limit mass for a neutron star(Kalogora 1996), where we assume the system consists of only two bodies. This has been the basis for BHC of Cyg X-1.

Black hole binaries exhibits characteristic X-ray properties such as distinctive spectral feature called "hard" or "soft" states. (Tanaka 1995). Although Cyg X-1 has been a prototypical blackhole candidate, it has rarely been found in "soft" states. In 1996 May, the RXTE/ASM reported an X- 


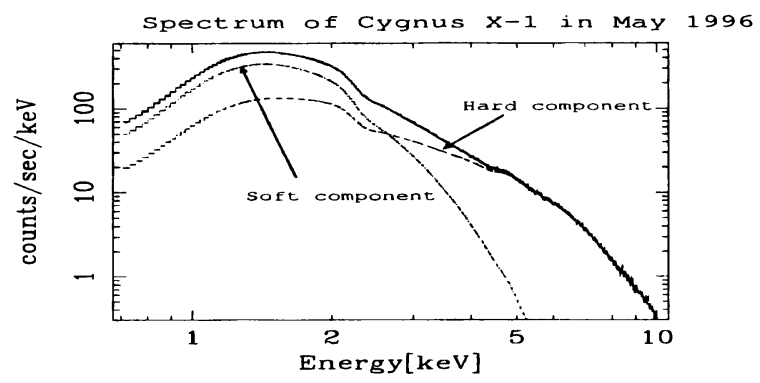

Figure 1. GIS2+GIS3

ray flare up of Cygnus X-1. We observed it with ASCA on 30-31 May for $33 \mathrm{ksec}$. The observed $0.7-10 \mathrm{keV}$ flux was $1.6 \times 10^{-8} \mathrm{erg} / \mathrm{cm}^{2} / \mathrm{sec}$. The obtained spectrum consists of the "Soft" and "Hard" components (Figure 1). The Soft component of the spectrum was well fitted with the multi-color accretion disk model (MCD model)(Mitsuda 1984) and the general relativistic accretion disk model(GRAD model)(hanawa 1989,ebisawa 1991) as observed in some other BHCs in the Soft states. A color temperature at the disk inner edge is $\sim 0.43 \mathrm{keV}$, and a disk bolometric flux is $\sim 5.60 \times 10^{-8}$ $\mathrm{erg} / \mathrm{sec} / \mathrm{cm}^{2}$. Assuming the distance of $2.5 \mathrm{kpc}$, the inclination of $30^{\circ}$, and the color-to-effective temperature ratio of 1.7 , and considering the inner boundary condition, the disk inner radius has been determined to be $\sim 90$ $\mathrm{km}$.

Assuming the Schwarzshild black hole,the inner edge of the accretion disk is thought to extend down to three times the Schwarzshild radius $\left(3 R_{\mathrm{s}}\right)$, which corresponds to the last stable orbit around the non-rotating black hole. By equationg the disk inner radius to $3 R_{\mathrm{s}}$, the mass of central object can be estimated.

Thus, we determined the mass of Cyg X-1 to be $12 \pm 2 M_{\odot}$. Application of the GRAD model gave a consistent result. These results confirm the existence of the black-hole in the Cygnus X-1 system.

\section{References}

Dotani et al. ApJL vol.485 in press

Oda, M. 1977, Space Sci. Rev. 20, 757

Liang, E. P. and Nolan, P. L. 1984, Space Sci. Rev. 38, 353

Herrero, A., Kudritzki, R. P., Gabler, R., Vilchez, J. M., Gabler, A. 1995, AAP 297, 556

Kalogera, V. and Baym, G. 1996, ApJL 470, L61

Tanaka, Y., and Lewin, W. H. G. 1995, X-Ray Binaries, eds. W. H. G. Lewin, J. van Paradijs \& W. P. J. van den Heuvel, (Cambridge University Press), 126

Makishima, K., Maejima, Y., Mitsuda, K. et al. 1986, PASJ 308, 635

Mitsuda K., Inoue, H., Koyama, K. et al. 1984, PASJ 36, 741

Hanawa T. 1989, ApJ 341, 948

Ebisawa, K., Mitsuda, K., Hanawa, T. 1991, ApJ 367, 213 